UCRHEP-T331

February 2002

\title{
Neutrinoless Double Beta Decay with Negligible Neutrino Mass
}

\author{
Biswajoy Brahmachari ${ }^{a}$ and Ernest $\mathrm{Ma}^{b}$ \\ (a) Theoretical Physics Group, Saha Institute of Nuclear Physics, \\ AF/1 Bidhannagar, Kolkata (Calcutta) 700064, INDIA \\ (b) Physics Department, University of California, \\ Riverside, California, CA 92521, USA
}

\begin{abstract}
$\underline{\text { Abstract }}$
If the electron neutrino has an effective nonzero Majorana mass, then neutrinoless double beta decay will occur. However, the latter is possible also with a negligible neutrino mass. We show how this may happen in a simple model of scalar diquarks and dileptons. This possibility allows neutrino masses to be small and hierarchical, without conflicting with the possible experimental evidence of neutrinoless double beta decay.
\end{abstract}


With the established evidence of atmospheric [1] and solar [2] neutrino oscillations, the notion of neutrino mass is generally accepted. Whereas there is yet no direct evidence of neutrino mass in beta decay [3], there is now a report [4] of the first positive evidence of neutrinoless double beta decay [5], which is commonly interpreted as being due to an effective nonzero Majorana mass of the electron neutrino. With this assumption, one may then explore the consequences [6] of having neutrino masses constrained by oscillations as well as neutrinoless double beta decay.

On the other hand, it is theoretically possible to have measurable neutrinoless double beta decay without having a corresponding Majorana neutrino mass of the expected magnitude [7]. In other words, the mechanism responsible for neutrinoless double beta decay may generate only a negligible Majorana neutrino mass. [Since lepton number is violated by two units, a nonzero Majorana neutrino mass is unavoidable, but it may be very small.]

Different mechanisms have been proposed in the past for contributions to neutrinoless double beta decay other than the electron neutrino Majorana mass. One possibility is to add a Higgs triplet $\left(h^{++}, h^{+}, h^{0}\right)$ in an $S U(2)_{L} \times U(1)_{Y}$ theory [8]. The couplings $W^{-} W^{-} h^{++}$ and $h^{++} e^{-} e^{-}$together can produce a quark-level diagram mimicking neutrinoless double beta decay. A second way is to embed the standard model in a left-right symmetric model [9]. In that case, $W_{R}$ exchange can also give rise to neutrinoless double beta decay. A combination of the right-handed neutrino Majorana mass and the $W_{R}$ mass scale is then constrained by experiment. In supersymmetric theories, the exchange of scalar quarks can mediate neutrinoless double beta decay if $R$-parity (lepton parity) is violated [10]. In this case, the diagram must include two $\lambda^{\prime}$ vertices, where each vertex violates lepton number by one unit. Vector-scalar contributions to neutrinoless double beta decay are also possible [11]. The lepton-number-violating vertex $u e^{-} \tilde{b}^{c}$ in supersymmetry together with $u d^{c} W^{-}$can induce neutrinoless double beta decay, whereas the vertices $u d^{c} \phi^{-}$and $u d^{c} W^{-}$may do the 
same in a left-right symmetric theory, with $\phi^{-}$coming from the $(2,2,0)$ representation.

Here we propose instead a simple model of scalar diquarks and dileptons [12] which has the following properties.

(1) Neutrinoless double beta decay occurs through the trilinear coupling of 2 scalar diquarks and 1 scalar dilepton.

(2) The Majorana neutrino mass generated by the above trilinear coupling occurs only in 4 loops and is negligibly small. [The dominant contributions to neutrino mass are assumed to come from some other mechanism not related to that of neutrinoless double beta decay.]

(3) The proposed scalar diquarks and dileptons interact only with first-generation fermions, i.e. $u, d$, and $e$. [This avoids constraints from $\mu \rightarrow e e e$ and $K^{0}-\overline{K^{0}}$ mixing, etc.]

(4) The smallness of $m_{u}, m_{d}$, and $m_{e}$ is understood in terms of a simple mechanism [13 in the Higgs sector.

(5) The model is verifiable experimentally at the TeV energy scale.

We now describe our model. Under $S U(3)_{C} \times S U(2)_{L} \times U(1)_{Y}$, the standard-model particle content is extended to include two scalar diquarks

$$
\Delta_{u} \sim(6,1,4 / 3), \quad \Delta_{d} \sim(6,1,-2 / 3)
$$

and one scalar dilepton

$$
\Delta_{e} \sim(1,1,-2)
$$

as well as a second Higgs doublet $\Phi_{2} \sim\left(\phi_{2}^{+}, \phi_{2}^{0}\right)$. We assume a discrete $Z_{3}$ symmetry $\left(\omega^{3}=1\right)$ under which

$$
d_{R}, e_{R}, \Delta_{u} \sim \omega, \quad u_{R}, \Delta_{d}, \Delta_{e}, \Phi_{2} \sim \omega^{2}
$$

and all other fields $\sim 1$. Thus the allowed Yukawa couplings are

$$
\Delta_{u}^{*} u_{R} u_{R}, \quad \Delta_{d}^{*} d_{R} d_{R}, \quad \Delta_{e}^{*} e_{R} e_{R}, \quad \overline{(u, d)}_{L} u_{R} \tilde{\Phi}_{2}, \quad \overline{(u, d)}_{L} d_{R} \Phi_{2}, \quad \overline{(\nu, e)}_{L} e_{R} \Phi_{2}
$$




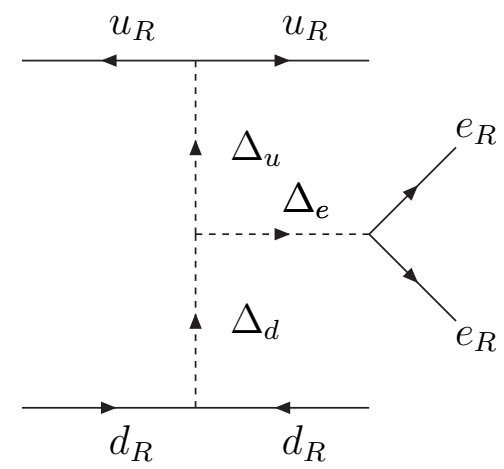

Figure 1: Diagram for neutrinoless double beta decay.

where $\tilde{\Phi}_{2} \equiv\left(\bar{\phi}_{2}^{0},-\phi_{1}^{-}\right)$. In the above, baryon number $(B)$ and lepton number $(L)$ are still conserved because $\Delta_{u}$ and $\Delta_{d}$ may be assigned $B=2 / 3$, and $\Delta_{e}$ may be assigned $L=2$. However, if the $Z_{3}$ symmetry is now assumed to be broken by the explicit soft terms $\Phi_{1}^{\dagger} \Phi_{2}$ and $\Delta_{u} \Delta_{d}^{*} \Delta_{e}$, then $B$ is still conserved but $L$ is broken down to $(-1)^{L}$, i.e. lepton parity.

The $\Phi_{1}^{\dagger} \Phi_{2}$ term allows $\phi_{2}^{0}$ to acquire a small vacuum expectation value naturally [13] so that the smallness of $m_{u}, m_{d}$, and $m_{e}$ may be understood. The other term violates lepton number by two units and may be responsible for neutrinoless double beta decay as shown in Fig. 1. Its amplitude is given by

$$
\mathcal{A}_{\beta \beta 0 \nu}=\frac{h_{u} h_{d} h_{e} m_{u d e}^{\text {soft }}}{m_{\Delta_{u}}^{2} m_{\Delta_{d}}^{2} m_{\Delta_{e}}^{2}},
$$

where $h_{u}, h_{d}, h_{e}$ are the dimensionless Yukawa couplings of $\Delta_{u}, \Delta_{d}, \Delta_{e}$ with $u, d, e$ pairs and $m_{\text {ude }}^{\text {soft }}$ is a soft mass term. As a crude estimate, if the effective Majorana mass of the electron neutrino is about $0.4 \mathrm{eV}$ [4], then $\mathcal{A}_{\beta \beta 0 \nu}$ should be about $10^{-16} \mathrm{GeV}^{-5}$ [7]. This may be satisfied for example with $h_{u}=h_{d}=h_{e}=1, m_{\Delta_{u}}=m_{\Delta_{d}}=m_{\Delta_{e}}=1 \mathrm{TeV}$, and $m_{\text {ude }}^{\text {soft }}=100$ GeV. From Fig. 1, it is also easy to see that its contribution to the electron-neutrino Majorana mass requires four loops, with two $\bar{u} d W^{+}$vertices, two $\bar{\nu} e W^{+}$vertices, and six helicity flips, which means that it is very much negligible. The origin of neutrino mass in this model is 
assumed to come from some other kind of physics, with negligible contribution to neutrinoless double beta decay.

We have assumed that baryon number is strictly conserved, but if that assumption is relaxed, then the soft term $\Delta_{u} \Delta_{d} \Delta_{d}$ is allowed and neutron-antineutron oscillation becomes possible. This has been discussed previously [14]. In our case, the discrete $Z_{3}$ symmetry also helps to suppress flavor-changing neutral currents, as discussed below.

Consider the most general scalar potential of the 2 assumed scalar doublets [13]:

$$
\begin{aligned}
V= & m_{1}^{2} \Phi_{1}^{\dagger} \Phi_{1}+m_{2}^{2} \Phi_{2}^{\dagger} \Phi_{2}+\frac{1}{2} \lambda_{1}\left(\Phi_{1}^{\dagger} \Phi_{1}\right)^{2}+\frac{1}{2} \lambda_{2}\left(\Phi_{2}^{\dagger} \Phi_{2}\right)^{2} \\
& +\lambda_{3}\left(\Phi_{1}^{\dagger} \Phi_{1}\right)\left(\Phi_{2}^{\dagger} \Phi_{2}\right)+\lambda_{4}\left(\Phi_{1}^{\dagger} \Phi_{2}\right)\left(\Phi_{2}^{\dagger} \Phi_{1}\right)+\left[\mu_{12}^{2} \Phi_{1}^{\dagger} \Phi_{2}+\text { h.c. }\right]
\end{aligned}
$$

where the $\mu_{12}^{2}$ term breaks the discrete $Z_{3}$ symmetry softly. The equations of constraint for $v_{1,2} \equiv\left\langle\phi_{1,2}^{0}\right\rangle$ are then

$$
\begin{aligned}
& v_{1}\left[m_{1}^{2}+\lambda_{1} v_{1}^{2}+\left(\lambda_{3}+\lambda_{4}\right) v_{2}^{2}\right]+\mu_{12}^{2} v_{2}=0 \\
& v_{2}\left[m_{2}^{2}+\lambda_{2} v_{2}^{2}+\left(\lambda_{3}+\lambda_{4}\right) v_{1}^{2}\right]+\mu_{12}^{2} v_{1}=0 .
\end{aligned}
$$

Let $m_{1}^{2}<0, m_{2}^{2}>0$, and $\left|\mu_{12}^{2}\right|<<m_{2}^{2}$, then

$$
v_{1}^{2} \simeq-\frac{m_{1}^{2}}{\lambda_{1}}, \quad v_{2}^{2} \simeq \frac{-\mu_{12}^{2} v_{1}}{m_{2}^{2}+\left(\lambda_{3}+\lambda_{4}\right) v_{1}^{2}} .
$$

Since the $\mu_{12}^{2}$ term breaks the $Z_{3}$ symmetry, it is natural [15] for it to be small compared to $m_{2}^{2}$. Thus $v_{2}<<v_{1}$ is obtained and since the first-generation quark and lepton masses are proportional to $v_{2}$, they are naturally small in this model.

The quark and lepton mass matrices $\left(\mathcal{M}_{u}, \mathcal{M}_{d}, \mathcal{M}_{e}\right)$ in this model are of the form

$$
\mathcal{M}=\left[\begin{array}{ccc}
f_{11} v_{2} & f_{12} v_{1} & f_{13} v_{1} \\
0 & f_{22} v_{1} & f_{23} v_{1} \\
0 & f_{32} v_{1} & f_{33} v_{1}
\end{array}\right]
$$


This means that flavor-changing neutral currents exist in the Higgs sector. However, they would be absent if $\Phi_{2}$ is replaced by $\Phi_{1}$ in Eq. (4). Hence the flavor-changing interactions must be contained in the terms

$$
f_{11}^{u} \bar{u}_{L} u_{R}\left(\overline{\phi_{2}^{0}}-\frac{v_{2}}{v_{1}} \overline{\phi_{1}^{0}}\right)+f_{11}^{d} \bar{d}_{L} d_{R}\left(\phi_{2}^{0}-\frac{v_{2}}{v_{1}} \phi_{1}^{0}\right)+f_{11}^{e} \bar{e}_{L} e_{R}\left(\phi_{2}^{0}-\frac{v_{2}}{v_{1}} \phi_{1}^{0}\right)+\text { h.c. },
$$

where $u_{L, R}$, etc. are in the basis of Eq. (10) and are not themselves mass eigenstates. The resultant off-diagonal terms are suppressed by the corresponding mixing angles and since $\phi_{2}^{0}$ is heavy and the coupling of $\phi_{1}^{0}$ is suppressed by $v_{2} / v_{1}$, the effect of flavor-changing neutral currents is very small in this model.

For illustration, consider the case of diagonal $\mathcal{M}_{d}$, then there are no tree-level flavorchanging interactions in the down sector, but since $\mathcal{M}_{u}$ must be rotated by the chargedcurrent mixing matrix $\left(V_{C K M}\right)$, flavor-changing interactions are unavoidable in the up sector. Allowing for the freedom to redefine $c_{R}$ and $t_{R}$, we can set $f_{32}=0$ for $\mathcal{M}_{u}$ in Eq. (10). Then

$$
m_{u} \simeq f_{11}^{u} v_{2}, \quad m_{c} \simeq f_{22}^{u} v_{1}, \quad m_{t} \simeq f_{33}^{u} v_{1}
$$

and the $\bar{u}_{L} u_{R}$ term in Eq. (11) becomes approximately [13]

$$
f_{11}^{u}\left(V_{u d}^{*} \bar{u}_{L}+V_{u s}^{*} \bar{c}_{L}+V_{u b}^{*} \bar{t}_{L}\right)\left(V_{u d} u_{R}+\frac{m_{u}}{m_{c}} V_{u s} c_{R}+\frac{m_{u}}{m_{t}} V_{u b} t_{R}\right)\left(\overline{\phi_{2}^{0}}-\frac{v_{2}}{v_{1}} \overline{\phi_{1}^{0}}\right)+\text { h.c. }
$$

in the mass-eigenvalue basis. This conributes to $D^{0}-\overline{D^{0}}$ mixing, i.e.

$$
\frac{\Delta m_{D^{0}}}{m_{D^{0}}} \simeq \frac{B_{D} f_{D}^{2} m_{u}^{3}}{3 m_{2}^{2} v_{2}^{2} m_{c}}\left|V_{u d}^{*} V_{u s}\right|^{2}
$$

Using $f_{D}=150 \mathrm{MeV}, B_{D}=0.8, m_{u}=4 \mathrm{MeV}, m_{c}=1.25 \mathrm{GeV},\left|V_{u d}\right| \simeq 1,\left|V_{u s}\right| \simeq 0.22$, and the experimental upper bound of $2.5 \times 10^{-14}$ [16], we find

$$
m_{2} v_{2}>24.4 \mathrm{GeV}^{2}
$$

which may be satisfied for example with $m_{2}=1 \mathrm{TeV}$ and $v_{2}=25 \mathrm{MeV}$. Another contribution to $D^{0}-\overline{D^{0}}$ mixing is through $\Delta_{u}$ exchange, i.e.

$$
\frac{\Delta m_{D^{0}}}{m_{D^{0}}} \simeq \frac{B_{D} f_{D}^{2} h_{u}^{2} m_{u}^{2}}{3 m_{\Delta_{u}}^{2} m_{c}^{2}}\left|V_{u d}^{*} V_{u s}\right|^{2} \simeq 3 \times 10^{-15}
$$


for $h_{u}=1$ and $m_{\Delta_{u}}=1 \mathrm{TeV}$, which is well below the present experimental upper bound.

At the TeV energy scale, the new scalars $\left(\Delta_{u}, \Delta_{d}, \Delta_{e}\right.$, and $\left.\Phi_{2}\right)$ of this model are expected to be produced in abundance, especially $\Delta_{u}$ and $\Delta_{d}$ at the LHC (Large Hadron Collider). The decays of $\Delta_{u, d}$ into two jets should provide a clear signature for their discovery. For the production of $\Delta_{e}$, the best accelerator would be an $e^{-} e^{-}$collider. It may also be produced in pairs at twice the energy at an $e^{+} e^{-}$collider, i.e. $e^{+} e^{-} \rightarrow \Delta_{e}^{*} \Delta_{e}$. Of course, the $e^{+} e^{-} \rightarrow e^{+} e^{-}$ cross section is also modified through its exchange.

In conclusion, new physics at the $\mathrm{TeV}$ scale may be responsible for a measurable Majorana mass of the electron neutrino without requiring the near mass-degeneracy of all three neutrinos. We have proposed a simple specific model of scalar diquarks and dileptons which allow this to happen, and is consistent with the present experimental bounds on flavor-changing neutral currents and other rare processes.

This work was supported in part by the U. S. Department of Energy under Grant No. DEFG03-94ER40837. 


\section{References}

[1] S. Fukuda et al., Super-Kamiokande Collaboration, Phys. Rev. Lett. 85, 3999 (2000) and references therein.

[2] S. Fukuda et al., Super-Kamiokande Collaboration, Phys. Rev. Lett. 86, 5656 (2001) and references therein. See also Q. R. Ahmad et al., SNO Collaboration, Phys. Rev. Lett. 87, 071301 (2001).

[3] J. Bonn et al., Nucl. Phys. Proc. Suppl. 91, 273 (2001); V. M. Lobashev et al., ibid. 91, 280 (2001).

[4] H. V. Klapdor-Kleingrothaus, A. Dietz, H. L. Harney, and I. V. Krivosheina, Mod. Phys. Lett. A16, 2409 (2001). However, this has been disputed by C. E. Aalseth et al., hep-ex/0202018.

[5] H. V. Klapdor-Kleingrothaus et al., Eur. Phys. J. A12, 147 (2001).

[6] Recent papers include H. V. Klapdor-Kleingrothaus and U. Sarkar, Mod. Phys. Lett. A16, 2469 (2001); hep-ph/0201226; hep-ph/0202006; E. Ma, hep-ph/0201225; V. Barger, S. L. Glashow, D. Marfatia, and K. Whisnant, hep-ph/0201262; F. Feruglio, A. Strumia, and F. Vissani, hep-ph/0201291; T. Hambye, hep-ph/0201307; H. Minakata, and H. Sugiyama, hep-ph/0202003; Z.-Z. Xing, hep-ph/0202034; N. Haba and T. Suzuki, hep-ph/0202143.

[7] For a recent review, see for example R. N. Mohapatra, hep-ph/9808284.

[8] J. Schechter and J. W. F. Valle, Phys. Rev. D22, 2227 (1980); D25, 2951 (1982); R. N. Mohapatra and J. D. Vergados, Phys. Rev. Lett. 47, 1713 (1981); L. Wolfenstein, Phys. Rev. D26, 2507 (1982). 
[9] Riazuddin, R. E. Marshak, and R. N. Mohapatra, Phys. Rev. D24, 1310 (1981).

[10] R. N. Mohapatra, Phys. Rev. D34, 3457 (1986).

[11] K. S. Babu and R. N. Mohapatra, Phys. Rev. Lett. 75, 2276 (1995).

[12] For general comprehensive studies of scalar bilinears, see E. Ma, M. Raidal, and U. Sarkar, Eur. Phys. J. C8, 301 (1999); Phys. Rev. D60, 076005 (1999).

[13] E. Ma, Phys. Rev. Lett. 86, 2502 (2001); Phys. Lett. B516, 165 (2001); Phys. Rev. D64, 097302 (2001).

[14] J. F. Nieves, Phys. Lett. B164, 85 (1985).

[15] G. 't Hooft, in Recent Developments in Gauge Theories: Proceedings of the NATO Advanced Study Institute (Cargese, 1979), edited by G. 't Hooft et al., (Plenum, New York, 1980).

[16] Particle Data Group, D. E. Groom et al., Eur. Phys. J. C15, 1 (2000). 